\title{
Genetic research in autism spectrum disorders
}

\author{
Elise B. Robinson ${ }^{\mathrm{a}, \mathrm{b}}$, Benjamin M. Neale $\mathrm{a}^{\mathrm{a}, \mathrm{b}}$, and Steven E. Hyman ${ }^{\mathrm{b}, \mathrm{c}}$
}

\begin{abstract}
Purpose of review
The recent explosion of genetic findings in autism spectrum disorder (ASD) research has improved knowledge of the disorder's underlying biology and etiologic architecture. This review introduces concepts and results from recent genetic studies and discusses the manner in which those findings can influence the trajectory of ASD research.
\end{abstract}

\begin{abstract}
Recent findings
Large consortium studies have associated ASDs with many types of genetic risk factors, including common polygenic risk, de novo single nucleotide variants, copy number variants, and rare inherited variants. In aggregate, these results confirm the heterogeneity and complexity of ASDs. The rare variant findings in particular point to genes and pathways that begin to bridge the gap between behavior and biology.
\end{abstract}

\section{Summary}

Genetic studies have the potential to identify the biological underpinnings of ASDs and other neuropsychiatric disorders. The data they generate are already being used to examine disease pathways and pathogenesis. The results also speak to ASD heterogeneity and, in the future, may be used to stratify research studies and treatment trials.

\section{Keywords}

autism, exome sequencing, genetics, GWAS

\section{INTRODUCTION}

Autism spectrum disorders (ASDs) are serious neuropsychiatric conditions characterized by impairments in social and communication behavior, as well as restricted and repetitive interests. Approximately, $1 \%$ of children in the United States meet the current diagnostic criteria for ASDs, with onset of symptoms in early childhood and persistence through the life course. Although the degree of impairment associated with ASDs varies substantially, affected individuals and their families are confronted by social, educational, and occupational challenges, for which treatment options are limited. The current treatments available for ASDs are predominantly behavioral, and have a limited impact on core behavioral symptomology. No pharmacologic treatments have been empirically associated with a reduction in autism symptoms or their frequent cognitive comorbidities. Unmet treatment need typifies neuropsychiatric disorders and reflects limited knowledge about the biological processes that drive pathogenesis.

Twin studies - notwithstanding some quantitative disagreements - have demonstrated significant heritability of ASDs, thus motivating large-scale genetic studies $\left[1,2^{-"}\right]$. Such studies aim to identify genetic variants that contribute to the risk of ASDs; genetic variants (alleles) represent DNA sequences that differ among individuals at specific genomic locations (loci). Depending on their genomic region, DNA sequence variants may influence the risk of an ASD by altering the amino acid sequence of a functional RNA or protein or by producing a change in the timing, circumstances, or level at which these molecules are expressed. A central goal of genetic research in ASDs and other neuropsychiatric disorders is to nominate molecular pathways for further interrogation and thus to link biological mechanisms with behavior. On the basis of the experience of other fields of medicine, the resulting knowledge should enable the discovery of new

\footnotetext{
${ }^{a}$ Analytic and Translational Genetics Unit, Department of Medicine, Massachusetts General Hospital and Harvard Medical School, Boston, ${ }^{b}$ Stanley Center for Psychiatric Research and Program in Medical and Population Genetics, Broad Institute of Harvard and MIT and 'Department of Stem Cell and Regenerative Biology, Harvard University, Cambridge, Massachusetts, USA

Correspondence to Elise B. Robinson, Stanley Center for Psychiatric Research, Broad Institute, 75 Ames Street, Cambridge, MA 02142, USA. Tel: +1 617714 7543; fax: +1617800 1764;

e-mail: erob@broadinstitute.org
}

Curr Opin Pediatr 2015, 27:685-691

DOI:10.1097/MOP.0000000000000278

This is an open access article distributed under the Creative Commons Attribution License 4.0, which permits unrestricted use, distribution, and reproduction in any medium, provided the original work is properly cited. 


\section{KEY POINTS}

- A series of recent consortium studies have associated ASDs with many types of genetic risk factors. These projects have greatly improved the understanding of the etiologic architecture of ASDs.

- ASDs are heterogeneous and complex, and genetic data can be used to better understand differences among affected individuals.

- Genetic findings are pointing to genes and pathways that begin to bridge the gap between behavior and biology.

treatments. Recent large consortial studies have identified the first genes that are convincingly associated with ASDs and have produced some early biological insights $\left[3^{-"}, 4^{-"}\right]$. These initial successes have also underscored the need for far larger sample sizes if we are to successfully elucidate the genetic architecture of ASDs. This review will highlight the goals and primary outcomes of recent ASD genetic studies, and discuss ways in which those data are being used to guide autism research.

\section{GENETIC INFLUENCES ON AUTISM SPECTRUM DISORDER RISK}

Genetic variants differ in nature and in the frequency at which they occur in the human population (Fig. 1). Inherited genetic variants, those that are passed from parents to children, can occur at all frequencies from common to very rare, whereas de novo variants, those that are newly arising in offspring and are not seen in a carrier's parents, are typically rare. Genetic studies have strongly suggested that the variant classes contributing to ASD risk are of all structural types, all frequencies, and can be inherited or de novo.

\section{Common polygenic variation}

Autism spectrum disorders are highly familial. In the United States, the siblings of children with ASDs are estimated to be at more than a 10-fold increase in risk for an ASD diagnosis themselves [5-8]. Moreover, the family members of children with ASDs are also more likely to have a history of psychiatric diseases defined broadly, including schizophrenia, bipolar disorder, and depression [9]. Such familial aggregation of psychiatric illness is consistent with complex, inherited genetic risk. Genome-wide association studies (GWAS) make it possible to identify common variants contributing to such polygenic risk by the use of DNA microarrays to detect common single nucleotide polymorphism (SNPs).

Methods for estimating heritability from SNP data - for example Genome-wide Complex Trait Analysis and LD (linkage disequilibrium) score regression $\left[10,11^{\prime}\right]$ - have permitted existing ASD GWAS collections to report strong, cumulative contributions of common inherited variants to autism risk. Across several recent studies, common genotyped SNPs are estimated to account for between $20 \%$ and $50 \%$ of variation in liability to ASDs $\left[2{ }^{*}, 11^{\prime}, 12\right]$. These estimates are consistent with those for other highly heritable, polygenic neuropsychiatric disorders, and predict that GWAS studies in ASDs will become an increasingly productive source of biological insight as sample size grows. ASD GWAS studies have yet to reliably nominate any specific loci but, as highlighted by the recent extraordinary successes in schizophrenia genetics [13"], psychiatric disease GWAS require very large case and control collections. As ASD sample collection efforts progress and GWAS studies become adequately powered, SNPs with significant association to ASDs will be identified, laying the foundation for follow-up biological interrogation.

Polygenic scoring and SNP heritability methods, both derived from GWAS, have also been used to identify genetic correlations between ASDs and other neuropsychiatric disorders, such as schizophrenia [12,14]. The genetic correlation between autism and schizophrenia is estimated to be approximately $20 \%$ using multiple genome-wide approaches. Overlapping genetic risk is a theme common to neuropsychiatric phenotypes, strongly suggesting that the biology underlying psychiatric disorders is unlikely to adhere to the diagnostic boundaries set out in such traditional classifications as the DSM-5 $\left[15-18,19^{-2}, 20\right]$.

\section{De novo variation}

The contribution of de novo (i.e., newly arising) variation to ASD risk has been identified through whole exome sequencing - the deep characterization of protein coding regions, which in aggregate comprise $1-2 \%$ of the genome [21-23]. The largest trio sequencing studies of ASDs published to date represent a clear advance in neurodevelopmental genomics, identifying a significant excess of functional mutations in more than 40 genes $\left[3^{\boldsymbol{*}}, 4^{\boldsymbol{*}}\right]$.

Although most people carry at least one de novo mutation somewhere in their exome [24], a de novo mutation at any given position is rare. The rarity of these events in specific genes means that it is necessary to build models to assess the significance of gene-disease relationships, even in the context of 


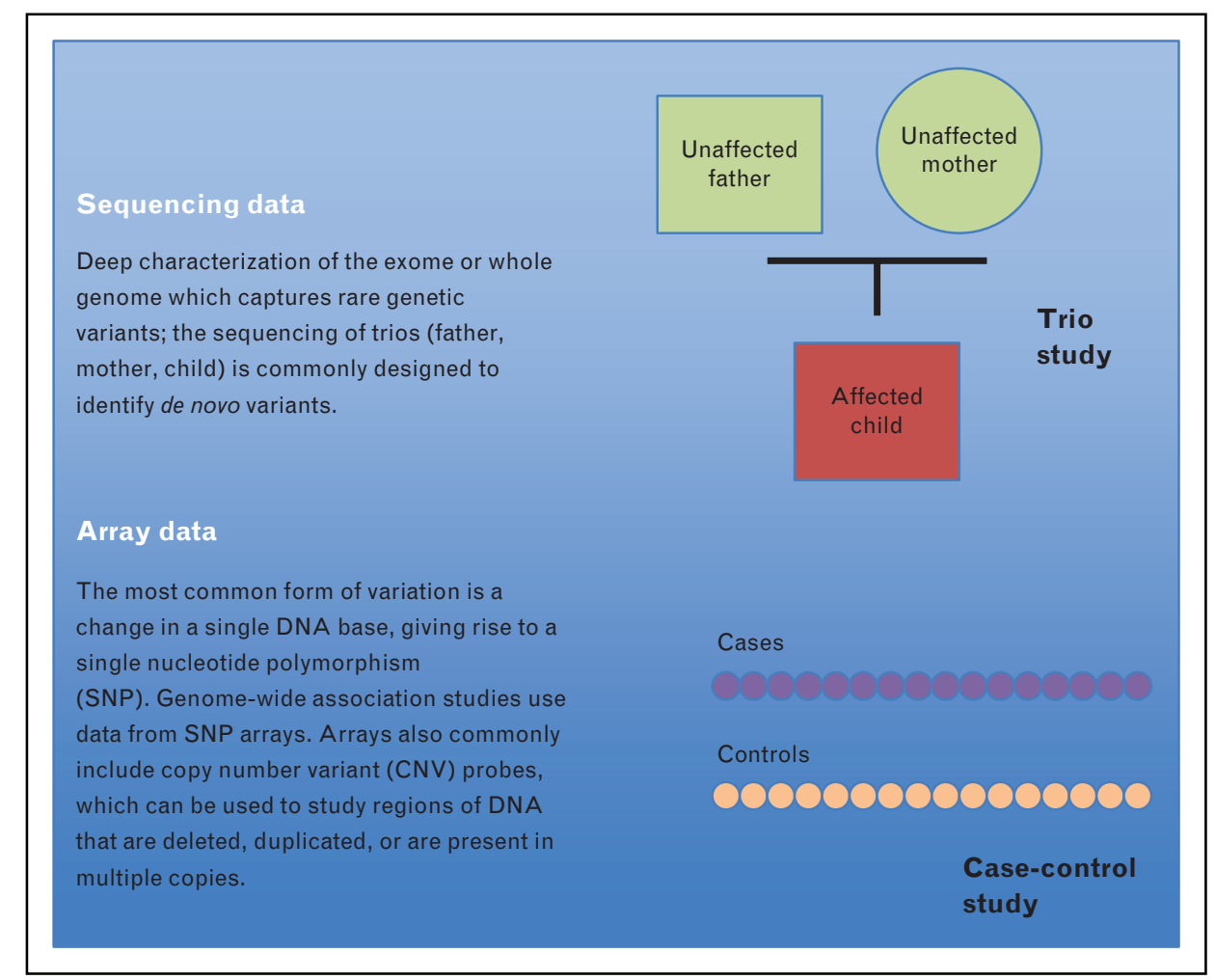

FIGURE 1. Study designs in autism spectrum disorders genetics.

genes in which multiple de novo mutations have been found in ASD cases. One such modeling approach estimates the mutation rate of variants of a given functional class (e.g., synonymous, missense) at a gene level, which can then be used to calculate the probability of observing a given number of mutations in the sample size in question [19"].

Statistical models can also be used to assess whether there is enrichment of certain functional classes of variants genome wide. For example, de novo loss of function (LoF) variants - de novo variants that result in loss of gene function - are seen in approximately $9 \%$ of people in the general population, and $16-18 \%$ of people with an ASD diagnosis $\left[3^{-"}, 4^{\prime \prime}\right]$. This suggests that de novo LoFs, on average, approximately double an individual's ASD risk, increasing the probability of being diagnosed with an ASD from approximately $1 \%$ to approximately $2 \%$. Individuals with ASDs also manifest a significant excess of de novo missense mutations, but the risk conferred by them is, on average, smaller. This level of enrichment suggests that additional genes will be significantly associated with ASDs as sequencing activities grow [3"',4"'].

On the basis of these results, recent studies have estimated that approximately 3 to $10 \%$ of ASD risk is attributable to de novo single-nucleotide variation in the exome $\left[2^{-\cdot}, 3^{\mathbf{*}}, 25^{\boldsymbol{\prime}}\right]$. The current meta-analytic picture reflects a significant but limited contribution that, as with GWAS associations, can be used to link behavior and biology. GWAS associations, however, identify a locus rather than a causal variant; moreover, approximately 90\% of GWAS associations across diseases are located within noncoding regions of the genome. Clearly identifiable mutations in protein-coding regions represent a more tractable situation for biological experimentation, given the broader toolkit currently available for interrogating protein function rather than gene regulation. The genes that have been strongly linked with ASDs in trio sequencing studies are associated with diverse biological functions [4"'], including both neuronal function and development processes. As a class, genes associated with ASDs show clear evidence of evolutionary constraint, as they are deficient in functional mutations in the population [19"].

Similar efforts at the association of de novo mutations with intellectual disability (ID) have identified more than 50 genes as significant contributors to risk [26"]. The great majority of those genes have also been implicated in ASD [3"',4"'], suggesting limited phenotypic specificity among neurodevelopmentally sensitive genes. 


\section{Copy number variants and inherited rare variation}

Copy number variants (CNVs) comprise the most common form of structural variation in the genome, and can be inherited or de novo. As with de novo SNVs, significant enrichment in the rate of de novo CNVs is observed in individuals with ASDs. Furthermore, CNVs in specific regions of the genome are associated with increased risk of ASDs [27]. Regionspecific CNV analyses were some of the first to consistently tie locations in the genome to ASD risk [17]. As discussed below, several specific CNVs that have been associated with ASDs are now being targeted for biological and phenotypic study.

An inherited rare variation, which can be investigated through both trio sequencing and the sequencing of cases and controls, is among the most difficult type of event to interrogate given the combination of low frequency and, on average, small effect size [28]. ASDs have been associated with inherited, two-hit (recessive) loss of function mutations and, more recently, a global excess of rare inherited protein-truncating variants in genes that are intolerant of mutation $\left[25^{-*}, 29\right]$. It is likely that additional rare inherited variant types will be associated with ASDs as sequencing studies increase in sample size and new techniques are developed to filter and highlight classes of variation most likely to be deleterious.

Risk factors for ASDs can be found across the full spectrum of genetic variation. Such a diverse landscape of genetic risk clearly demonstrates that ASDs are a polygenic trait, with a myriad of different risk factors in the population. These genetic clues are the first step in gaining insight into the biological underpinnings of ASDs.

\section{HETEROGENEITY IN AUTISM SPECTRUM DISORDERS - GENOTYPE TO PHENOTYPE}

The recent explosion of genetic associations in ASD research has highlighted the diversity of the disorder's etiologic influences. The phenotypic heterogeneity of ASDs has been recognized far longer, and several avenues of research now aim to associate genetic risk factors for ASDs with specific phenotypic differences.

The prevalence of individuals diagnosed with ASDs has increased rapidly over the past 20 years. Despite concerns that higher prevalence might suggest new pathogenic environmental factors, the majority of the increase appears to reflect diagnostic expansion - both diagnostic substitution between ID and ASDs and, to an even greater degree, diagnostic extension into the high functioning range [30-32]. The most recent US population surveys suggest that the ID rate in ASD has dropped by 50\% in only 25 years, indicating a striking shift in diagnostic practice and the average phenotypic profile of those diagnosed [33]. Lower intelligence quotient (IQ) in ASDs is correlated with increased behavioral symptom severity and an increased rate of comorbid epilepsy and other neurological symptoms. Thus, an increase in mean IQ predicts higher functioning across a variety of phenotypic dimensions [34"].

Several 'genotype to phenotype' relationships in ASDs have recently been examined $\left[3^{-*}, 34^{\prime \prime}, 35,36\right]$, with the association between de novo mutations and proband IQ emerging as particularly significant. Specifically, the rate of de novo LoFs in low IQ ASDs substantially exceeds that seen in high IQ ASDs. The association between IQ and de novo LoFs in the Simons Simplex Collection is shown in Fig. 2. The LoF rate in ASDs crosses the rate seen in other disorders at several points along the IQ distribution. At very low IQ, the LoF rate is similar to that seen in samples ascertained for severe ID [19"]. In high IQ groups, it intersects with the average rates seen in people with schizophrenia $[37,38]$ and the rates seen in healthy control populations.

Some of this trend is likely to reflect a general relationship between cognition and rare, damaging mutations in the population as a whole, in both those with and without diagnosable neuropsychiatric disorders. To the extent that differences in genetic architecture in ASDs, however, predict differences in underlying biology, clinical features, or treatment response, such genotype-phenotype relationships warrant further exploration, whether starting with phenotypes or genotypes. For example, recent studies have been conditioned on specific individual genes and CNVs, such that the spectrum of biological and clinical findings associated with those genomic events can be better characterized [39"',40,41"]. The Simons Variation in Individuals Project is one such experiment. The investigators have collected extensive behavioral, cognitive, and biological data from several hundred carriers of an ASD-associated $\mathrm{CNV}$, typically a duplication or deletion at a particular chromosomal locus, $16 \mathrm{p} 11.2$, as well as the carriers' family members. The size and diversity of the data set permits consideration of several questions that cannot be addressed in a study of individuals ascertained only for ASDs. For example, MorenoDe-Luca et al. [39"'] recently reported an association between parental cognitive and behavioral profiles and phenotypic variation in carriers of de novo 16p11.2 deletions, strongly suggesting an additive relationship between inherited polygenic and de novo risk on neuropsychiatric phenotypes. Another recent study carefully examined the phenotype associated with mutations in the CHD8 gene, which has been 


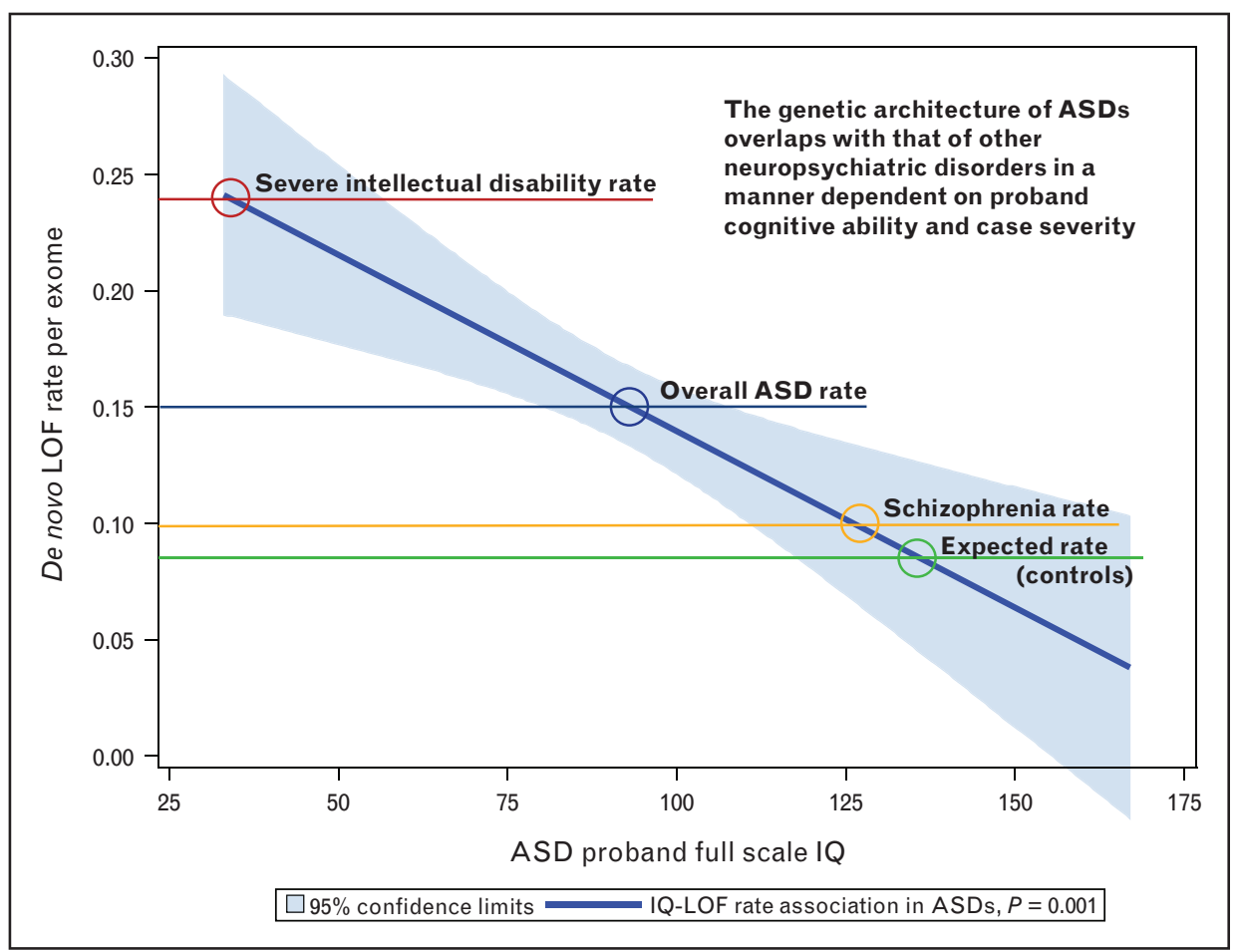

FIGURE 2. Loss of function (LoF) regression generated using data from the Simons Simplex Collection as described by Robinson et al. [34"]; LoF rate for severe intellectual disability and controls from Samocha et al. [19"]; LoF rate for schizophrenia from Fromer et al. [38]. ASD, autism spectrum disorder; IQ, intelligence quotient.

associated with ASDs in multiple exome sequencing studies [3"-41",42]. Compared with other individuals with ASDs, CHD8 carriers were more likely to have macrocephaly and gastrointestinal symptoms, along with other distinguishing features.

A limit to the 'genotype first' line of inquiry may come from the limited number of genes, or the limited number of recurrent CNVs, that exert a strong enough influence on ASD risk and are seen in more than a handful of cases. As described above, the average odds ratio for ASDs associated with de novo LoF mutations is approximately 2 . There is likely to be substantially variability surrounding that average [4"'], but one can use it to estimate limits on the fraction of variants within the class that confer very strong ASD risk. For example, using an odds ratio of 2 , fewer than $5 \%$ of de novo LoF variants could have an ASD odds ratio of 20 before having to postulate that the remaining $95 \%$ of de novo LoFs variants are collectively protective against autism. CHD8 is likely to be an uncommonly affected gene with a large odds ratio and as such it is very well chosen to begin the search for genetically defined ASD subtypes. Other syndromic forms of ASDs (e.g., Fragile X, 22q11.2 deletion syndrome) are also more likely to have common phenotypic presentations, but as the landscape of contributing variation continues to be uncovered, it is likely that most ASD cases will be complex (i.e., cannot be strongly associated with any given genetic or environmental risk factor).

The complexity of most cases will similarly place limits on the diagnostic yield of clinical sequencing and genotyping panels. Families and clinicians have become increasingly interested in associating individual ASD cases with genetic risk factors. Although a small percentage of ASD cases will carry a clearly established, large ASD risk factor, the majority of returns from such efforts are unlikely to be easily interpreted. For the limited subset of families whose affected child carries an established risk factor - for example a de novo LoF mutation in CHD8 - the return from clinical sequencing could be emotionally valuable and could meaningfully inform the parents' concept of familial ASD risk. However, the great majority of de novo or inherited events detected through clinical panels will have made a very small contribution, if any contribution at all, to the development of ASD in a given case. The interpretation of the information returned must then be cautious. In most ASD patients in whom a de novo LoF mutation or other event type is found, the majority of the genetic contribution to that patient is still likely to be inherited. In other words, if the average de novo LoF mutation doubles ASD risk (from approximately $1 \%$ to $2 \%$ ), about $98 \%$ of individual liability is left to be explained, and familial factors are likely to be the largest remaining 
contributors [2"']. The clear communication of clinical sequencing results to families will substantially affect the value of those activities.

\section{CONCLUSION}

As with other studies of neuropsychiatric traits, genetic studies of ASDs have enormous potential to point to the biology underlying behavior. In addition to aiding in the discovery and development of therapeutics by nominating molecular targets and suggesting new hypotheses for biomarkers, a more complete picture of genetic architecture could lead to the productive stratification of epidemiologic studies and clinical trials. Particularly with continued aggregation of samples in consortium studies, genomics will greatly improve the understanding of ASD biology in the coming years, and may contribute significantly to much needed new treatments.

\section{Acknowledgements}

We thank Rosy Hosking for her help in editing the manuscript.

\section{Financial support and sponsorship}

E.B.R. was funded by National Institute of Mental Health Grant 1K01MH099286-02. S.E.H is supported by a gift from the Stanley Foundation. B.M.N. was supported by National Institute of Mental Health Grant 5U01MH094432-04.

\section{Conflicts of interest}

S.E.H. is a principal investigator on a collaboration with Novartis Institutes for Biomedical Research, and has advised Novartis, AstraZeneca, and Sunovion on early drug discovery during the past three years.

\section{REFERENCES AND RECOMMENDED \\ READING}

Papers of particular interest, published within the annual period of review, have been highlighted as:

- of special interest

- of outstanding interest

1. Ronald A, Hoekstra RA. Autism spectrum disorders and autistic traits: a decade of new twin studies. Am J Med Genet B Neuropsychiatr Genet 2011; 156:255-274

2. Gaugler T, Klei L, Sanders SJ, et al. Most genetic risk for autism resides with - common variation. Nat Genet 2014; 46:881-885.

This study reported that most of the genetic influences on ASD risk reflect common, inherited variation. They estimate the proportion of ASD risk attributable to multiple types of genetic variants and find that rare de novo events can have large individual effects but have a limited contribution to risk at a population level. 3. lossifov I, O'Roak BJ, Sanders SJ, et al. The contribution of de-novo coding -1 mutations to autism spectrum disorder. Nature 2014; 515:216-221.

This study, copublished with De Rubeis et al., uses large-scale whole-exome sequencing technology to examine the contribution of inherited and germline de novo mutations to ASD risk. The authors identified high-confidence gene targets and suggested that de novo missense mutations contribute to a significant number of diagnoses.
4. De Rubeis $S, H e X$, Goldberg $A P$, et al. Synaptic, transcriptional and I. chromatin genes disrupted in autism. Nature 2014; 515:209-215.

This study, copublished with lossifov et al., uses large-scale whole-exome sequencing technology to examine the contribution of inherited and germline de novo mutations to ASD risk. The authors identified hundreds of de novo LoF mutations, which were estimated to contribute to ASD risk in more than $5 \%$ of individuals with autism. The genes harboring de novo LoF mutations were reported to affect synaptic, transcriptional, and chromatin remodeling pathways.

5. Risch N, Hoffmann TJ, Anderson M, et al. Familial recurrence of autism spectrum disorder: evaluating genetic and environmental contributions. Am J Psychiatry 2014; 171:1206-1213.

6. Ozonoff S, Young GS, Carter A, et al. Recurrence risk for autism spectrum disorders: a Baby Siblings Research Consortium study. Pediatrics 2011; 128:e488-e495.

7. Constantino JN, Todorov A, Hilton C, et al. Autism recurrence in half siblings: strong support for genetic mechanisms of transmission in ASD. Mol Psychiatry 2013; 18:137-138.

8. Constantino JN, Zhang Y, Frazier T, et al. Sibling recurrence and the genetic epidemiology of autism. Am J Psychiatry 2010; 167:1349-1356.

9. Sullivan PF, Magnusson C, Reichenberg A, et al. Family history of schizophrenia and bipolar disorder as risk factors for autism. Arch Gen Psychiatry 2012; 69:1099-1103.

10. Yang J, Lee SH, Goddard ME, Visscher PM. GCTA: a tool for genome-wide complex trait analysis. Am J Hum Genet 2011; 88:76-82.

11. Bulik-Sullivan BK, Loh $P R$, Finucane HK, et al. LD score regression distin-

- guishes confounding from polygenicity in genome-wide association studies. Nat Genet 2015; 47:291-295.

This study describes a statistical approach for distinguishing between polygenicity and confounding in genome-wide association studies. This method also provides an opportunity to estimate the heritability of a trait using GWAS summary statistics.

12. Lee SH, Ripke S, Neale BM, et al. Genetic relationship between five psychiatric disorders estimated from genome-wide SNPs. Nat Genet 2013; 45:984-994.

13. Schizophrenia Working Group of the Psychiatric Genomics Consortium.

- Biological insights from 108 schizophrenia-associated genetic loci. Nature 2014; 511:421-427.

This meta-analysis used data from more than 50 studies, totaling over 30000 patients, to conduct a genome-wide association study of schizophrenia. The GWAS identified 108 independent genetic loci associated with schizophrenia risk, and is the most productive common variant study in neuropsychiatric disease to date.

14. Bulik-Sullivan BK, Finucane HK, Anttila V, et al. An atlas of genetic correlations across human diseases and traits. bioRxiv 2015; doi: http://dx.doi.org/ 10.1101/014498. [Epub ahead of print]

15. Smoller JW, Craddock N, Kendler K, et al. Identification of risk loci with shared effects on five major psychiatric disorders: a genome-wide analysis. Lancet 2013; 381:1371-1379.

16. Sebat J, Levy DL, McCarthy SE. Rare structural variants in schizophrenia: one disorder, multiple mutations; one mutation, multiple disorders. Trends Genet 2009; 25:528-535.

17. Weiss LA, Shen $Y$, Korn JM, et al. Association between microdeletion and microduplication at 16p11.2 and autism. N Engl J Med 2008; 358:667-675.

18. McCarthy SE, Makarov V, Kirov G, et al. Microduplications of $16 \mathrm{p} 11.2$ are associated with schizophrenia. Nat Genet 2009; 41:1223-1227.

19. Samocha KE, Robinson EB, Sanders SJ, et al. A framework for the inter- pretation of de novo mutation in human disease. Nat Genet 2014.

This study introduced a statistical model for association in de novo variant studies. The model can be used to estimate the probability with which a gene or gene set is associated with a disease.

20. Sullivan PF, Daly MJ, O'Donovan M. Genetic architectures of psychiatric disorders: the emerging picture and its implications. Nat Rev Genet 2012; 13:537-551.

21. lossifov I, Ronemus $M$, Levy $D$, et al. De-novo gene disruptions in children on the autistic spectrum. Neuron 2012; 74:285-299.

22. Sanders SJ, Murtha MT, Gupta AR, et al. De-novo mutations revealed by whole-exome sequencing are strongly associated with autism. Nature 2012; 485:237-241.

23. Neale BM, Kou Y, Liu L, et al. Patterns and rates of exonic de novo mutations in autism spectrum disorders. Nature 2012; 485:242-245.

24. Kong $A$, Frigge ML, Masson $G$, et al. Rate of de novo mutations and the importance of father's age to disease risk. Nature 2012; 488:471-475.

25. Krumm N, Turner $\mathrm{TN}$, Baker $\mathrm{C}$, et al. Excess of rare, inherited truncating mutations in autism. Nat Genet 2015; 47:582-588.

This study analyzes multiple classes of rare variation in the exome, and estimates the ASD risk attributable to each class. The authors identify a subset of rare, inherited mutations that increase ASD risk.

26. Name M, Fitzgerald TW, Gerety SS, Jones WD, et al. Deciphering develop-

- mental disorders large-scale discovery of novel genetic causes of developmental disorders. Nature 2015; 519:223-228.

The Deciphering Developmental Disorders project is the largest trio sequencing project to date focused on ID. The study identified over 50 genes that create risk for ID. 
27. Sanders SJ, Ercan-Sencicek AG, Hus V, et al. Multiple recurrent de novo CNVs, including duplications of the $7 q 11.23$ Williams syndrome region, are strongly associated with autism. Neuron 2011; 70:863885

28. Stein JL, Parikshak NN, Geschwind DH. Rare inherited variation in autism: beginning to see the forest and a few trees. Neuron 2013; 77:209211.

29. Lim ET, Raychaudhuri S, Sanders SJ, et al. Rare complete knockouts in humans: population distribution and significant role in autism spectrum disorders. Neuron 2013; 77:235-242.

30. King $M$, Bearman $P$. Diagnostic change and the increased prevalence of autism. Int J Epidemiol 2009; 38:1224-1234.

31. Fombonne E. Epidemiological surveys of pervasive developmental disorders. In: Volkmar F, editor. Autism and pervasive developmental disoders. Cambridge, UK: Cambridge University Press; 2007.

32. Fombonne E. Epidemiology of autistic disorder and other pervasive developmental disorders. J Clin Psychiatry 2005; 66:3-8.

33. CDC (2014) Prevalence of autism spectrum disorders: Autism and Developmental Disabilities Monitoring Network, 11 sites, United States, 2010. MMWR Surveill Summ 63(2):1-22.

34. Robinson EB, Samocha KE, Kosmicki JA, et al. Autism spectrum disorder

- severity reflects the average contribution of de novo and familial influences. Proceedings of the National Academy of Sciences of the United States of America 2014.

This study associated IQ and other indicators of case severity in ASDs with differences in the disorders' genetic architecture. Compared with high IQ patients, ASD patients with low IQ were more likely to have de novo LoF mutations and less likely to have a family history of broadly defined psychiatric disease.
35. Chaste P, Klei L, Sanders SJ, et al. A genome-wide association study of autism using the Simons Simplex Collection: does reducing phenotypic heterogeneity in autism increase genetic homogeneity? Biol Psychiatry 2015; 77:775-784.

36. Ronemus $M$, lossifov I, Levy $D$, Wigler $M$. The role of de-novo mutations in the genetics of autism spectrum disorders. Nat Rev Genet 2014; 15:133-141.

37. Purcell SM, Moran JL, Fromer M, et al. A polygenic burden of rare disruptive mutations in schizophrenia. Nature 2014; 506:185-190.

38. Fromer $\mathrm{M}$, Pocklington $\mathrm{AJ}$, Kavanagh $\mathrm{DH}$, et al. De novo mutations in schizophrenia implicate synaptic networks. Nature 2014; 506:179-184.

39. Moreno-De-Luca A, Evans DW, Boomer KB, et al. The role of parental

- cognitive, behavioral, and motor profiles in clinical variability in individuals with chromosome 16p11.2 deletions. JAMA Psychiatry 2015; 72:119-126. This study examined the relationship between family background and phenotypic variation among carriers of de novo deletions at $16 \mathrm{p} 11.2$. The results strongly suggested an additive relationship between inherited polygenic and de novo risk on ASD and other neuropsychiatric phenotypes.

40. Hanson E, Bernier R, Porche K, et al. The cognitive and behavioral phenotype of the $16 \mathrm{p} 11.2$ deletion in a clinically ascertained population. Biol Psychiatry 2015; 77:785-793.

41. Bernier R, Golzio $C$, Xiong $B$, et al. Disruptive $C H D 8$ mutations define a - subtype of autism early in development. Cell 2014; 158:263-276.

This study provided an in-depth analysis of phenotypic profile in individuals with disruptive mutations in CHD8, a gene that is strongly associated with ASD risk. The results suggest that $C H D 8$ mutations define an ASD subtype and highlight comorbidities between brain and gastrointestinal development.

42. O'Roak BJ, Vives L, Girirajan S, et al. Sporadic autism exomes reveal a highly interconnected protein network of de-novo mutations. Nature 2012; 485:246250. 